\title{
Some Results on Output Algebraic Feedback with Applications to Mechanical Systems
}

\author{
F. Barbagli ${ }^{\dagger}$ \\ G. Marro ${ }^{\dagger}$ \\ P. Mercorelli ${ }^{\ddagger}$ \\ D. Prattichizzo ${ }^{\ddagger}$ \\ ${ }^{\dagger}$ Dipartimento di Elettronica, Informatica e Sistemistica, Università di Bologna, Italy \\ ‡Dipartimento di Ingegneria dell'Informazione, Università di Siena, Italy \\ gmarro@deis.unibo.it, mercorelli[prattichizzo]@ing.unisi.it
}

\begin{abstract}
Constructive necessary and sufficient conditions for disturbance decoupling with algebraic output feedback are presented. Necessary and sufficient conditions are also derived for the decoupling problem with internal stability. The same conditions are re-stated in terms of invariant zeros. The groundwork throughout is the duallattice structures of invariants introduced by Basile and Marro in [2]. Finally, an application to mechanical systems is presented.
\end{abstract}

\section{Introduction}

It is well known that disturbance decoupling was the first problem approached with geometric techniques, by Basile and Marro [1] and, independently, by Wonham and Morse [10]. In the former of these papers the investigation was extended to output feedback using a dynamic regulator. The same problem was refined in the literature by also taking into account the stability requirement (Willems and Commault [9] and, without using eigenspaces, Basile, Marro and Piazzi [3]). Anyway to the authors' knowledge the problems of disturbance decoupling and disturbance decoupling with internal stability using algebraic output feedback have not found a solution yet.

In this work, starting off from the necessary and sufficient conditions derived in the past we determine constructive necessary and sufficient conditions for the first problem and non-constructive necessary and sufficient conditions for the second one by using the geometric approach tools. The same conditions will be also derived by using the concept of invariant zeros first introduced by Rosenbrock [8].

The following notation is used. $\mathbf{R}$ stands for the field of real numbers. Sets, vector spaces and subspaces are denoted by script capitals like $\mathcal{X}, \mathcal{I}, \mathcal{V}$, etc.; since most of the geometric theory of dynamic system herein presented is developed in the vector space $\mathbf{R}^{n}$, we reserve the symbol $\mathcal{X}$ for the full space, i.e., we assume $\mathcal{X}:=\mathbf{R}^{n}$. The orthogonal complement of any subspace $\mathcal{Y} \subseteq \mathcal{X}$ is denoted by $\mathcal{Y}^{\perp}$, matrices and linear maps by slanted capitals like $A, B$, etc., the image and the null space of the generic matrix or linear transformation $A$ by $\operatorname{im} A$ and $\operatorname{ker} A$ respectively, the transpose of the generic real matrix $A$ by $A^{T}$, the spectrum of $A$ by $\sigma(A)$, the $n \times n$ identity matrix by $I_{n}$. The restriction of map $A$ to the $A$-invariant subspace $\mathcal{L}$ is denoted by $\left.A\right|_{\mathcal{L}}$. Given two $A$-invariants $\mathcal{L}_{1}$ and $\mathcal{L}_{2}$ such that $\mathcal{L}_{1} \subseteq \mathcal{L}_{2}$, the map induced by $A$ on the quotient space $\mathcal{L}_{1} / \mathcal{L}_{2}$ is denoted by $\left.A\right|_{\mathcal{L}_{1} / \mathcal{L}_{2}}$. Notation $\mathcal{Z}_{1}-\mathcal{Z}_{2}$ will be used for the difference of sets $\mathcal{Z}_{1}$ and $\mathcal{Z}_{2}$ with repetition count.

\section{Background and problem statement}

Let us consider a system described by a five-map system $(A, B, C, D, E)$, modeled by

$$
\begin{aligned}
& \dot{x}(t)=A x(t)+B u(t)+D d(t), \quad x(0)=x_{0} \\
& y(t)=C x(t) \\
& e(t)=E x(t)
\end{aligned}
$$

where $x \in \mathcal{X}\left(=\mathbf{R}^{n}\right), u \in \mathcal{U}\left(=\mathbf{R}^{p}\right), d \in \mathcal{D}\left(=\mathbf{R}^{d}\right), e \in \mathcal{E}$ $\left(=\mathbf{R}^{m}\right)$ and $y \in \mathcal{Y}\left(=\mathbf{R}^{q}\right)$ denote respectively the state, the manipulable input, the disturbance, the regulated output and the informative output. In the following the short notations $\mathcal{B}:=\operatorname{im} B, \mathcal{C}:=\operatorname{ker} C, \mathcal{D}:=\operatorname{im} D$ and $\mathcal{E}:=\operatorname{ker} E$ will be used. The problem of simple disturbance decoupling by means of output algebraic feedback is stated as follows:

Problem 1. Given the system (1)-(3) determine, if possible, a feedback matrix $K$ (having $p$ rows and $q$ columns) such that:

i) $e(t)=0, t \geq 0$, for all admissible $d($.$) and for$ $x(0)=0$.

The problem of disturbance decoupling with stability by means of output algebraic feedback is stated as:

Problem 2. Assume for system (1)-(3) that $(A, B)$ is stabilizable and $(A, C)$ detectable. Determine, if possible, a feedback matrix $K$ (having $p$ rows and $q$ columns) such that:

i) Problem 1 is solvable; 
ii) $\lim _{t \rightarrow \infty} x(t)=0$, for all $x(0)$ with $d()=$.0 .

Conditions (i) and (ii) are called respectively the structure requirement and the stability requirement.

The following theorem introduced by Basile and Marro [2] (pag. 256) is basic to solve Problem 1:

Theorem 1. Refer to triple $(A, B, C)$. There exists a matrix $K$ such that a given subspace $\mathcal{V}$ is an $(A+B K C)$-invariant if and only if $\mathcal{V}$ is both an $(A, \mathcal{B})$ controlled and an $(A, \mathcal{C})$-conditioned invariant.

This theorem is constructive, i.e., given such a subspace $\mathcal{V}$ there exists a procedure to determine matrix $K$.

Let us recall now the definitions of lattices $\phi(\mathcal{B}+\mathcal{D}, \mathcal{E})$ and $\psi(\mathcal{C} \cap \mathcal{E}, \mathcal{D})$ on which the next considerations will be based:

$$
\begin{aligned}
\phi(\mathcal{B}+\mathcal{D}, \mathcal{E}) & :=\{\mathcal{V} \mid \\
A \mathcal{V} \subseteq \mathcal{V} & \left.+\mathcal{B}+\mathcal{D}, \mathcal{V} \subseteq \mathcal{E}, \mathcal{V}^{*} \cap(\mathcal{B}+\mathcal{D}) \subseteq \mathcal{V}\right\}
\end{aligned}
$$

is the lattice of all $(A, \mathcal{B}+\mathcal{D})$-controlled invariants self bounded with respect to $\mathcal{E}$, and its supremum and infimum are given by

$$
\begin{aligned}
\mathcal{V}^{*} & :=\max \mathcal{V}(A, \mathcal{B}+\mathcal{D}, \mathcal{E}) \\
\mathcal{V}_{m} & :=\mathcal{V}^{*} \cap \min \mathcal{S}(A, \mathcal{E}, \mathcal{B}+\mathcal{D})
\end{aligned}
$$

respectively, while

$\psi(\mathcal{C} \cap \mathcal{E}, \mathcal{D}):=\{\mathcal{S} \mid$

$$
\left.A(\mathcal{S} \cap \mathcal{C} \cap \mathcal{E}) \subseteq \mathcal{S}, \mathcal{D} \subseteq \mathcal{S}, \mathcal{S} \subseteq \mathcal{S}^{*}+(\mathcal{C} \cap \mathcal{E})\right\}
$$

is the lattice of all $(A, \mathcal{C} \cap \mathcal{E})$-conditioned invariants self hidden with respect to $\mathcal{D}$, with infimum and supremum given by

$$
\begin{aligned}
\mathcal{S}^{*} & :=\min \mathcal{S}(A, \mathcal{C} \cap \mathcal{E}, \mathcal{D}) \\
\mathcal{S}_{M} & :=\mathcal{S}^{*}+\max \mathcal{V}(A, \mathcal{D}, \mathcal{C} \cap \mathcal{E}) .
\end{aligned}
$$

All of the above subspaces are easily determined through the standard geometric approach algorithms.

Finally Let us recall the definitions of left and right invertibility. Under the assumption that $B$ and $C$ have maximum rank, the triple $(A, B, C)$ is said to be:

$$
\begin{aligned}
& \text { left-invertible if and only if } \mathcal{V}_{0}^{*} \cap \mathcal{B}=\emptyset \text {, with } \\
& \mathcal{V}_{0}^{*}:=\max \mathcal{V}(A, \mathcal{B}, \mathcal{C}) ; \\
& \text { right-invertible if and only if } \mathcal{S}_{0}^{*}+\mathcal{C}=\mathbf{R}^{n} \text {, with } \\
& \mathcal{S}_{0}^{*}:=\min \mathcal{S}(A, \mathcal{C}, \mathcal{B}) \text {. }
\end{aligned}
$$

\section{Structural Conditions}

Let us consider Problem 1. Clearly the problem admits solution if and only if the reachable set by $d$, i.e. the minimal $(A+B K C)$-invariant containing $\mathcal{D}$ is contained in $\mathcal{E}$. By Theorem 1 , Problem 1 is solvable if and only if there exists a subspace $\mathcal{V}$ so that:

$$
\begin{aligned}
& \text { i) } \mathcal{D} \subseteq \mathcal{V} \subseteq \mathcal{E} \\
& \text { ii) } \mathcal{V} \text { is an }(A, \mathcal{B}) \text { - controlled invariant } \\
& \text { iii ) } \mathcal{V} \text { is an }(A, \mathcal{C}) \text { - conditioned invariant }
\end{aligned}
$$

Necessary but not sufficient conditions for the existence of such a $\mathcal{V}$ are:

$$
\begin{aligned}
& \mathcal{D} \subseteq \mathcal{V}^{*} \\
& \mathcal{S}^{*} \subseteq \mathcal{E} \\
& \mathcal{S}^{*} \subseteq \mathcal{V}^{*}
\end{aligned}
$$

The proof of the above conditions is trivial. Conditions (13) and (14) derive from (10) while (15) derives from (11)-(12).

Under assumptions (13)-(15) some very interesting properties regarding lattices (4) and (7) can be determined. Under assumption (13) it can be proved that

i) every subspace of lattice (4) contains $\mathcal{D}$,

ii) $\mathcal{V}^{*}=\max \mathcal{V}(A, \mathcal{B}, \mathcal{E})$

while under (14)

i) every subspace of lattice (7) is contained in $\mathcal{E}$,

ii) $\mathcal{S}^{*}=\min \mathcal{S}(A, \mathcal{C}, \mathcal{D})$,

as seen in [2] (pag. 225-226).

Two very useful sublattices of (4) and (7), introduced in [2] (pag. 271) are:

$$
\begin{aligned}
& \phi_{R}:=\left\{\mathcal{V} \mid \mathcal{V} \in \phi(\mathcal{B}+\mathcal{D}, \mathcal{E}), \mathcal{V}_{m} \subseteq \mathcal{V} \subseteq \mathcal{V}_{M}\right\} \\
& \psi_{R}:=\left\{\mathcal{S} \mid \mathcal{S} \in \psi(\mathcal{C} \cap \mathcal{E}, \mathcal{D}), \mathcal{S}_{m} \subseteq \mathcal{S} \subseteq \mathcal{S}_{M}\right\}
\end{aligned}
$$

where $V_{m}$ and $S_{M}$ are given by (6) and (9) and, under assumptions (13)-(15),

$$
\begin{aligned}
& \mathcal{V}_{M}:=\mathcal{V}_{m}+\mathcal{S}_{M}, \\
& \mathcal{S}_{m}:=\mathcal{V}_{m} \cap \mathcal{S}_{M} .
\end{aligned}
$$

From now on conditions (13)-(15) will be considered automatically satisfied. Using the sublattices the first result is stated.

Theorem 2. Referring to (1)-(3), Problem 1 is solvable if relation

$$
\mathcal{V}_{m} \subseteq \mathcal{S}_{M}
$$

holds.

Proof: Subspaces $\mathcal{V}_{m}$ and $\mathcal{S}_{M}$ are both solutions of the problem under assumption (20). In fact $\mathcal{V}_{m}$ satisfies (10)-(11), being the infimimum of $\phi(\mathcal{B}+\mathcal{D}, \mathcal{E})$, and (12) since

$$
\mathcal{S}_{m}:=\mathcal{V}_{m} \cap \mathcal{S}_{M}=\mathcal{V}_{m} \Rightarrow \mathcal{V}_{m} \in \psi(\mathcal{C} \cap \mathcal{E}, \mathcal{D})
$$


Dually $S_{M}$ satisfies (10) and (12), being the supremum of $\psi(\mathcal{C} \cap \mathcal{E}, \mathcal{D})$, and $(11)$ since

$$
\mathcal{V}_{M}:=\mathcal{V}_{m}+\mathcal{S}_{M}=\mathcal{S}_{M} \Rightarrow \mathcal{S}_{M} \in \phi(\mathcal{B}+\mathcal{D}, \mathcal{E})
$$

This very interesting result has been derived without any assumption on the system's invertibility. Unfortunately condition (20) is only sufficient. It becomes both necessary and sufficient if the system in hand is both left and right-invertible, as stated in the following:

Theorem 3. Let the given system be both leftinvertible with respect to $u$ and right-invertible with respect to $y$. Problem 1 is solvable if and only if relation (20) holds.

Proof: (Only if) Being the system left and rightinvertible all $(A, \mathcal{B})$-controlled invariant subspaces are also self bounded with respect to $\mathcal{E}$ and all $(A, \mathcal{C})$ conditioned invariant subspaces are also self hidden with respect to $\mathcal{D}$. This means that any subspace solving the problem, i.e. satisfying conditions (10)-(12), must be an element of both $\phi(\mathcal{B}+\mathcal{D}, \mathcal{E})$ and $\psi(\mathcal{C} \cap \mathcal{E}, \mathcal{D})$. Clearly if relation (20) is not satisfied then lattices (4) and (7) have no intersection and so the problem has no solution.

(If) Implied by Theorem 2 .

It is important to note that hardly the systems in hand are both left and right-invertible. If one of these assumption fails relation (20) is not necessary anymore. Anyway we are able to state new necessary and sufficient conditions for the solvability of the problem:

Theorem 4. Let the given system be left-invertible with respect to input $u$. Problem 1 is solvable if and only if $\mathcal{V}_{m}$ is an $(A, \mathcal{C})$-conditioned invariant.

Proof: (Only if) By the left invertibility assumption $\mathcal{V}^{*} \cap \mathcal{B}=\emptyset$. This means that every $(A, \mathcal{B})$-controlled invariant is self bounded with respect to $\mathcal{E}$. This means that a subspace $\mathcal{V}$ satisfying properties (10)-(12), has to be searched for in $\phi(\mathcal{B}+\mathcal{D}, \mathcal{E})$. We want to show that if such a $\mathcal{V}$ exists then $\mathcal{V}_{m}$ is an $(A, \mathcal{C})$-conditioned invariant. Being $\mathcal{V}$ an element of $\phi(\mathcal{B}+\mathcal{D}, \mathcal{E})$, hence $\mathcal{V}_{m} \subseteq \mathcal{V}$, it follows that

$$
\left(\mathcal{V}_{m} \cap \mathcal{C}\right) \subseteq(\mathcal{V} \cap \mathcal{C}) \Rightarrow A\left(\mathcal{V}_{m} \cap \mathcal{C}\right) \subseteq A(\mathcal{V} \cap \mathcal{C})
$$

and, being $\mathcal{V}$ an $(A, \mathcal{C})$-conditioned invariant,

$$
A\left(\mathcal{V}_{m} \cap \mathcal{C}\right) \subseteq A(\mathcal{V} \cap \mathcal{C}) \subseteq \mathcal{V}
$$

On the other hand, $\mathcal{V}_{m}$ being an $(A, \mathcal{B})$-controlled invariant implies that

$$
A \mathcal{V}_{m} \subseteq \mathcal{V}_{m}+\mathcal{B} \Rightarrow A\left(\mathcal{V}_{m} \cap \mathcal{C}\right) \subseteq \mathcal{V}_{m}+\mathcal{B}
$$

The two relations above show that $A\left(\mathcal{V}_{m} \cap \mathcal{C}\right)$ is included in the intersection of subspaces $\mathcal{V}$ and $\mathcal{V}_{m}+\mathcal{B}$, hence

$$
\begin{aligned}
& A\left(\mathcal{V}_{m} \cap \mathcal{C}\right) \subseteq \\
& \left(\mathcal{V}_{m}+\mathcal{B}\right) \cap \mathcal{V}=\left(\mathcal{V} \cap \mathcal{V}_{m}\right)+(\mathcal{B} \cap \mathcal{V})=\mathcal{V}_{m}
\end{aligned}
$$

since $\mathcal{V}_{m} \subseteq \mathcal{V}$ and since $\mathcal{V}^{*} \cap \mathcal{B}=\emptyset \Rightarrow \mathcal{V} \cap \mathcal{B}=\emptyset$.

(If) Obvious by virtue of (10)-(12).

Corollary 1. Let the given system be right-invertible with respect to output $y$. The disturbance decoupling problem with algebraic output feedback is solvable if and only if $\mathcal{S}_{M}$ is an $(A, \mathcal{B})$-controlled invariant.

Proof: Dual to proof of Theorem 4.

All of these conditions are easily checkable through appropriate algorithms and are constructive, meaning that, when a subspace satisfying Theorem 1 has been determined, it is easy to obtain a matrix $K$ solving the problem.

The following decomposition, which can be applied to a system satisfying statements (13)-(15), is very useful to prove the next statements.

Decomposition 1. Let us assume the similarity transformation $T:=\left[\begin{array}{lllll}T_{1} & T_{2} & T_{3} & T_{4} & T_{5} \\ T_{6}\end{array}\right]$, with $\operatorname{im} T_{1}=S^{*}:=$ $\min \mathcal{S}(A, \mathcal{C}, \mathcal{D}), \operatorname{im}\left[T_{1} T_{2}\right]=\mathcal{S}_{M} \cap \mathcal{V}_{m}, \operatorname{im}\left[T_{1} T_{2} T_{3}\right]=\mathcal{S}_{M}$, $\operatorname{im}\left[\begin{array}{lll}T_{1} & T_{2} & T_{4}\end{array}\right]=\mathcal{V}_{m}, \operatorname{im}\left[\begin{array}{lllll}T_{1} & T_{2} & T_{3} & T_{4} & T_{5}\end{array}\right]=\mathcal{V}^{*}=$ $\max \mathcal{V}(A, \mathcal{B}, \mathcal{E})$ and $T_{6}$ such that $T$ is nonsingular. In the new basis matrices $A, B, C, D$ and $E$ are expressed by

$$
\begin{aligned}
& A^{\prime}=\left[\begin{array}{cccccc}
A_{11}^{\prime} & A_{12}^{\prime} & A_{13}^{\prime} & A_{14}^{\prime} & A_{15}^{\prime} & A_{16}^{\prime} \\
A_{21}^{\prime} & A_{22}^{\prime} & A_{23}^{\prime} & A_{24}^{\prime} & A_{25}^{\prime} & A_{26}^{\prime} \\
O & O & A_{33}^{\prime} & O & A_{53}^{\prime} & A_{56}^{\prime} \\
A_{41}^{\prime} & O & O & A_{44}^{\prime} & A_{45}^{\prime} & A_{46}^{\prime} \\
O & O & O & O & A_{55}^{\prime} & A_{56}^{\prime} \\
A_{61}^{\prime} & O & O & A_{64}^{\prime} & A_{65}^{\prime} & A_{66}^{\prime}
\end{array}\right] \\
& B^{\prime}=\left[\begin{array}{c}
B_{1}^{\prime} \\
B_{2}^{\prime} \\
O \\
B_{4}^{\prime} \\
O \\
B_{6}^{\prime}
\end{array}\right], D^{\prime}=\left[\begin{array}{c}
D_{1}^{\prime} \\
O \\
O \\
O \\
O \\
O
\end{array}\right] \\
& C^{\prime}=\left[\begin{array}{llllll}
C_{1}^{\prime} & O & O & C_{4}^{\prime} & C_{5}^{\prime} & C_{6}^{\prime}
\end{array}\right], \\
& E^{\prime}=\left[\begin{array}{llllll}
O & O & O & O & O & E_{6}^{\prime}
\end{array}\right] .
\end{aligned}
$$

Note that this representation has been obtained without any assumptions on either left or right invertibility for the system.

This decomposition becomes more simple if the system is left or right-invertible. In the former case matrices $B_{1}^{\prime}, B_{2}^{\prime}$ and $B_{4}^{\prime}$ are zero while in the latter matrices $C_{4}^{\prime}, C_{5}^{\prime}$ and $C_{6}^{\prime}$ are zero. Using this decomposition we derived the following Corollary (proved in [5]).

Corollary 2. Let the system be SISO and either left or right-invertible. Problem 1 has in general no solution. 
This is a clear example of how regulators using algebraic output feedback work. For the disturbance decoupling to have a solution using this kind of feedback it is obviously necessary that the same problem has a solution through state feedback, but this is not sufficient. In fact it is also necessary to use "enough" outputs to evaluate "enough" state variables, since through algebraic output feedback we have no information on $x$. SISO systems, in general, have too few outputs to solve the problem using this kind of regulator. Clearly, extending the rank of matrix $C$, which on a practical level means using more sensors, increases knowledge of state $x$ and consequently the chances that Problem 1 is solvable.

In the most general case of a system being neither left nor right-invertible we are only able to state a constructive sufficient condition as seen in Theorem 2. Anyway if relation (20) does not hold, as it often happens, the following result is very useful for the search of a resolvent:

Property 1. Problem 1 admits a solution iff

i) $\mathcal{V}_{m}$ is an $(A, \mathcal{C})$-conditioned invariant, or

ii) $\mathcal{S}_{M}$ is an $(A, \mathcal{B})$-controlled invariant, or

iii) a subspace $\mathcal{V}$ being both an $(A, \mathcal{B})$-controlled invariant and an $(A, \mathcal{C})$-conditioned invariant exists such that $\mathcal{S}_{m} \subset \mathcal{V} \subset \mathcal{V}_{m}$ or $\mathcal{S}_{M} \subset \mathcal{V} \subset \mathcal{V}_{M}$.

Proof: (Only if) Suppose that a solution $\mathcal{V}$ exists such that $\mathcal{S}^{*} \subseteq \mathcal{V} \subseteq \mathcal{S}_{M} \cap \mathcal{V}_{m}$. In such case it is possible to extend Decomposition 1 by choosing $T_{1}=\left[\begin{array}{ll}T_{11} & T_{12}\end{array}\right]$ with $\operatorname{im}\left(T_{11}\right)=S^{*}$ and $\operatorname{im}\left(T_{1}\right)=\mathcal{V}$. For $\mathcal{V}$ to be a solution of Problem 1 there must exist a $K$ such that $A_{41_{1}}^{\prime}+B_{4}^{\prime} K C_{1_{1}}^{\prime}=0, A_{61_{1}}^{\prime}+B_{6}^{\prime} K C_{1_{1}}^{\prime}=0$, $A_{41_{2}}^{\prime}+B_{4}^{\prime} K C_{1_{2}}^{\prime}=0$ and $A_{61_{2}}^{\prime}+B_{6}^{\prime} K C_{1_{2}}^{\prime}=0$. So matrix $K$ solves the problem also for $S_{M}$. The same considerations can be repeated if we suppose that a solution $\mathcal{V}$ exists such that $\mathcal{S}_{M} \cap \mathcal{V}_{m} \subseteq \mathcal{V} \subseteq \mathcal{S}_{M}$. Dual considerations can be made if the supposed solution $\mathcal{V}$ is such that $\mathcal{V}_{m} \subseteq \mathcal{V} \subseteq \mathcal{V}^{*}$.

If neither $\mathcal{V}_{m}$ nor $S_{M}$ are a solution for the problem the subspace that solves it has to be looked for in a "narrower" space, but we can't state if that subspace actually exists or not and we have no procedure to determine it. Anyway in many practical cases it has been shown that subspace $\mathcal{V}_{m}$ solves the problem.

\section{Disturbance Decoupling with Stability}

Let us consider now Problem 2. The following holds:

Theorem 5. Let the given system be left-invertible with respect to input $u$ and and the pair $(A, B)$ be stabilizable. Problem 2 is solvable if and only if:

i) $\mathcal{V}_{m}$ is an $(A, \mathcal{C})$-conditioned invariant; ii) $\mathcal{V}_{M}$ is internally stabilizable;

iii) $\exists F \mid(A+B F) \mathcal{V} \subseteq \mathcal{V},(A+B F)_{\mathcal{X} / \mathcal{V}}$ is stable, $\operatorname{ker} C \subseteq \operatorname{ker} F$

Proof: Let us consider Decomposition 1 with $B^{\prime}$ having the particular structure seen for left-invertible systems. Obviously the first condition is still necessary but not sufficient anymore. This is due to the fact that we are looking for a matrix $K$ so that $\mathcal{V}_{m}$ is an $(A+B K C)$ invariant and so that matrix $(A+B K C)$ has all eigenvalues stable. Clearly if the former condition is verified then matrix $(A+B K C)$ has a triangular structure so that $\sigma(A+B K C)=\sigma\left(A_{1}\right) \cup \sigma\left(A_{2}\right)$

where

$$
\begin{gathered}
A_{1}:=\left[\begin{array}{cccc}
A_{11}^{\prime} & A_{12}^{\prime} & A_{13}^{\prime} & A_{14} \\
A_{21}^{\prime} & A_{22}^{\prime} & A_{23}^{\prime} & A_{24}^{\prime} \\
O & O & A_{33}^{\prime} & O \\
A_{41}^{\prime} & O & O & A_{44}^{\prime}
\end{array}\right] \\
A_{2}:=\left[\begin{array}{cc}
A_{55}^{\prime} & A_{56}^{\prime} \\
A_{65}^{\prime}+B_{6}^{\prime} K C_{5}^{\prime} & A_{66}^{\prime}+B_{6}^{\prime} K C_{6}^{\prime}
\end{array}\right] .
\end{gathered}
$$

The first set of eigenvalues is stable if and only if relation (ii) holds while the second one is stable if and only if:

1. the system is stabilizable so that $\mathcal{V}_{M}$ is externally stabilizable as an $(A, \mathcal{B})$-controlled invariant i.e. $\exists F \mid \quad(A+B F) \mathcal{V}_{M} \subseteq \mathcal{V}_{M},(A+B F)_{\mathcal{X} / \mathcal{V}_{M}}$ is stable;

2. there exists a matrix $K$ of output feedback which results perfectly mappable with one of the state feedback matrix satisfying condition 1 just mentioned i.e. given one of the above $F$, exists $K$ such that $F=K C \Leftrightarrow C^{T} K^{T}=F^{T} \Leftrightarrow \operatorname{im} F^{T} \subseteq$ $\operatorname{im} C^{T} \Leftrightarrow \operatorname{ker} C \subseteq \operatorname{ker} F$,

i.e. (iii) holds.

Corollary 3. Let the given system be right-invertible with respect to output $y$ and the pair $(A, C)$ be detectable. Problem 2 is solvable if and only if:

i) $\mathcal{S}_{M}$ is an $(A, \mathcal{B})$-controlled invariant

ii) subspace $\mathcal{S}_{m}$ is externally stabilizable

iii) $\exists G \mid(A+C G) \mathcal{S} \subseteq \mathcal{S}, \quad(A+C G)_{\mathcal{S}}$ is stable, $\operatorname{im} G \subseteq \operatorname{im} B$

Proof: Dual of proof to Theorem 5.

The same conditions can be stated in terms of invariant zeros. The following holds:

Theorem 6. Let the given system be left-invertible with respect to input $u$ and and the pair $(A, B)$ be stabilizable. Problem 2 is solvable if and only if: 
i) $\mathcal{V}_{m}$ is an $(A, \mathcal{C})$-conditioned invariant;

ii) $\mathcal{Z}(u ; e)-\mathcal{Z}(u, d ; e)$ are all stable

iii) $\mathcal{Z}(u, d ; e) \cap \mathcal{Z}(d ; y, e)$ are all stable

iv) $\exists F \mid(A+B F) \mathcal{V} \subseteq \mathcal{V},(A+B F)_{\mathcal{X} / \mathcal{V}}$ is stable, $\operatorname{ker} C \subseteq \operatorname{ker} F$

Proof: We want to show the equivalence of these conditions with the ones stated in Theorem 5 . The first and last conditions are the same for both theorems, so we just need to show that condition (ii) of Theorem 5 holds if and only if conditions (ii)-(iii) hold for Theorem 6 . Condition (ii) of Theorem 5 can be divided in two parts:

1. $\mathcal{V}_{m}$ is internally stabilizable,

2. submatrix $A_{33}^{\prime}$ has stable eigenvalues.

It has been shown in the past (by Piazzi and Marro [6]) that $\mathcal{V}_{m}$ is internally stabilizable if and only if $\mathcal{Z}(u ; e)-$ $\mathcal{Z}(u, d ; e)$ are all stable. It is easy to see that submatrix $A_{33}^{\prime}$ has stable eigenvalues if and only if $\mathcal{Z}(u, d ; e) \cap$ $\mathcal{Z}(d ; y, e)$ are all stable since

$$
\begin{aligned}
& \mathcal{Z}(u, d ; e)=\sigma\left(A_{33}^{\prime}\right) \cup \sigma\left(A_{55}^{\prime}\right) \\
& \mathcal{Z}(d ; y, e)=\sigma\left(A_{22}^{\prime}\right) \cup \sigma\left(A_{33}^{\prime}\right)
\end{aligned}
$$

and so the equivalence is proved.

Corollary 4. Let the given system be right-invertible with respect to input $y$ and and the pair $(A, C)$ be detectable. Problem 2 is solvable if and only if:

i) $\mathcal{S}_{M}$ is an $(A, \mathcal{B})$-controlled invariant;

ii) $\mathcal{Z}(d ; y)-\mathcal{Z}(d ; y, e)$ are all stable

iii) $\mathcal{Z}(u, d ; e) \cap \mathcal{Z}(d ; y, e)$ are all stable

iv) $\exists G \mid(A+C G) \mathcal{S} \subseteq \mathcal{S}, \quad(A+C G)_{\mathcal{S}}$ is stable, $\operatorname{im} G \subseteq \operatorname{im} B$

Proof: Dual to proof of Theorem 6.

\section{Applications to mechanical systems}

In order to both validate the theoretical results and show their potential applications to mechanical systems, the problem of controlling vehicles equipped with active suspensions is here stated in a geometric framework as a decoupling problem.

The control of active suspensions has been widely investigated in literature, e.g. [4]. Active suspensions are employed in advanced vehicles in order to enhance both ride comfort and safety. The actuation of suspensions along with proper sensor systems allows the vehicle controller to actively reject external disturbances.

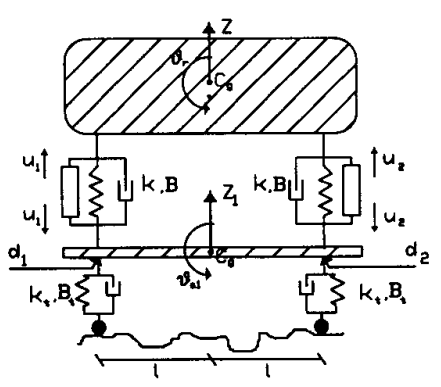

Figure 1: 2D mechanical model of a vehicle with active suspensions.

This section is aimed to synthesize a decoupling control law able to isolate the chassis from disturbances due to road irregularity and transmitted through the suspensions. This problem is usually referred to as ride height regulation.

\subsection{Dynamic model of the vehicle}

The two-dimensional vehicle in fig. 1 consists of a rigid chassis and a rigid axis linked by means of two passive suspensions and actuators. An independent control action is exerted at each corner of the vehicle $\left(u_{1}\right.$ and $\left.u_{2}\right)$.

With the notation of equations (1-3), a five map representation $(A, B, C, D, E)$ of the vehicle dynamics around the equilibrium configuration has been derived. The 8-dimensional state vector consists of the chassis roll $\theta_{r}$ and height $z$, of the wheel axis height $z_{1}$ and angle $\theta_{a 1}$ and their respective derivatives:

$$
\begin{gathered}
x=\left(\begin{array}{ll}
x_{r}^{T} & x_{v}^{T}
\end{array}\right)^{T} \\
x_{r}=\left(\begin{array}{llll}
\theta_{r} & \theta_{a 1} & \dot{\theta}_{r} \dot{\theta}_{a 1}
\end{array}\right)^{T}, \quad x_{v}=\left(\begin{array}{llll}
z & z_{1} & \dot{z} & \dot{z}_{1}
\end{array}\right)^{T} .
\end{gathered}
$$

We are interested in regulating, through control inputs $u=\left(u_{1}, u_{2}\right)^{T}$ and informative outputs $y$, the chassis posture $e=\left(\theta_{r}, z\right)^{T}$ against disturbances $d=\left(d_{1}, d_{2}\right)^{T}$.

According to [7], the state matrix is

$$
A=\left[\begin{array}{cc}
A_{11} & 0_{4} \\
0_{4} & A_{22}
\end{array}\right] \text { with } A_{i i}=\left[\begin{array}{cc}
0_{2} & I_{2} \\
M_{i k} & M_{i \beta}
\end{array}\right]
$$

$M_{1 k}=\left[\begin{array}{cc}\frac{-2 k l^{2}}{I_{r}} & \frac{2 k l^{2}}{I_{r}} \\ \frac{2 k l^{2}}{I_{a 1}} & \frac{-2\left(k_{t}+k\right) l^{2}}{I_{a 1}}\end{array}\right], \quad M_{2 k}=\left[\begin{array}{cc}\frac{-2 k}{M_{b}} & \frac{2 k}{M_{b}} \\ \frac{2 k}{M_{a 1}} & \frac{-2\left(k_{t}+k\right)}{M_{a 1}}\end{array}\right]$, to obtain $M_{1 \beta}$ and $M_{2 \beta}$ simply substitute $k$ with $\beta$; the input matrix is

$$
B=\left[\begin{array}{c}
B_{1} \\
B_{2}
\end{array}\right] \quad \text { with } B_{i}=\left[\begin{array}{c}
0_{2} \\
B_{i L}
\end{array}\right], B_{i L}=\left[\begin{array}{cc}
\frac{-l}{I_{T}} & \frac{l}{I_{r}} \\
\frac{l}{I_{a i}} & -\frac{l}{I_{a i}}
\end{array}\right]
$$

the disturbance matrix is

$$
D=\left[\begin{array}{l}
D_{1} \\
D_{2}
\end{array}\right] \quad \text { with } D_{i}=\left[\begin{array}{c}
0_{2} \\
D_{i L}
\end{array}\right], \quad D_{i L}=\left[\begin{array}{cc}
0 & 0 \\
\frac{-l}{I_{a i}} & \frac{l}{I_{a i}}
\end{array}\right]
$$

and the controlled output matrix is

$$
E=\left[\begin{array}{l|l|l|l}
1 \\
0
\end{array}\left|0_{(2 \times 3)}\right| \begin{array}{l}
0 \\
1
\end{array} \mid 0_{(2 \times 3)}\right]
$$


where $M_{b}\left(I_{r}\right)$ is the mass (moment of inertia) of the chassis (about the roll axis); $M_{a 1}\left(I_{a 1}\right)$ is the mass (inertia) of the axis with length $2 l ; k(\beta)$ is the spring (damping) coefficient of suspension and finally $k_{t}$ and $\beta_{t}$ are the visco-elastic parameters of the tires.

Assuming that the suspension heights and their time derivatives are accessible for measurements, the informative output vector is

$$
y=\left[y_{h}^{T}, \dot{y}_{h}^{T}\right]^{T}=C x
$$

where $y_{h}=\left[\begin{array}{l}\left(z-\theta_{r} l\right)-\left(z_{1}-\theta_{a 1} l\right) \\ \left(z+\theta_{r} l\right)-\left(z_{1}+\theta_{\mathbf{a} 1} l\right)\end{array}\right]$.

According to this formulation the problem of ride height regulation reduces to Problem 1 of Section 2 .

\subsection{Disturbance decoupling example}

A realistic example of a road vehicle with active suspensions is here reported. The used parameters of the vehicle geometry and dynamics are $l=0.9 \mathrm{~m} ; M_{b}=$ $1500 \mathrm{~kg} ; I_{r}=360 \mathrm{kgm}^{2} ; M_{a 1}=40 \mathrm{~kg} ; I_{a 1}=10.8 \mathrm{Kgm}^{2}$; $K=18 E 4 N / m ; \beta=1 E 3 N s / m ; K_{t}=1.96 E 5 N / m$; $\beta_{t}=1.92 E 3 \mathrm{Ns} / \mathrm{m}$.

Being $\max \mathcal{V}(A, \mathcal{B}, \mathcal{C}) \cap \mathcal{B}=\emptyset$, it results that the mechanical system is left-invertible with respect to the informative outputs $y$ and that the necessary and sufficient condition of Theorem 4 holds for

$$
V_{m}=\operatorname{im}\left[\begin{array}{llllllll}
0 & 1 & 0 & 0 & 0 & 0 & 0 & 0 \\
0 & 0 & 0 & 0 & 0 & 1 & 0 & 0 \\
0 & 0 & 0 & 1 & 0 & 0 & 0 & 0 \\
0 & 0 & 0 & 0 & 0 & 0 & 0 & 1
\end{array}\right]^{\mathrm{T}}
$$

The informative output feedback gain

$$
K=10^{4}\left[\begin{array}{rrll}
19 & 1 & 0.11 & 0.0056 \\
1 & 19 & 0.0056 & 0.11
\end{array}\right]
$$

localizes disturbances $d$ in the null space of the regulated output $e=\left(\theta_{r}, z\right)$. Geometrically, feedback gain $K$, makes the resolvent $V_{m}(A+B K C)$-invariant. As regards the decoupling problem with stability, stated in Problem 2, it can be easily shown that necessary conditions i) and ii) of Theorem 5 hold. Observe that in order to enjoy sufficiency of Theorem 5 condition iii), which strongly depends upon the specific control problem, should be met as well.

Note that results on disturbance localization for active suspensions are not just limited to the particular twodimensional example here reported. In [7] it has been proved that the unaccessible disturbance localization for the regulated output $e$ can be viewed as a structural property of all the vehicles equipped with active suspensions.

\section{Conclusions}

A solution for the problem of disturbance decoupling using algebraic output feedback has been considered.
The necessary and sufficient conditions for the structural problem (without stability) are easily checkable and constructive. For the problem with stability requirement the conditions are not constructive anymore: the solution has to be searched among the output-toinput matrices solving the structural problem. The structural part of the problem may have no solution, or only one solution, in which case we have no freedom on choosing matrix $K$ so that the final system is stable, or more solutions, giving us a chance to look for a matrix $K$ solving the problem with stability.

In practical applications, the solution of the disturbance decoupling problem through algebraic output feedback strongly simplifies the structure of the control systems and enhances the whole system robustness. This has been put into evidence by the potential application of paper results to some mechanical systems. In particular, the problem of controlling vehicles equipped with active suspensions has been formalized as a decoupling problem and solved in a geometric framework.

\section{References}

[1] G. Basile and G. Marro, "L'invarianza rispetto ai disturbi studiata nello spazio degli stati," Rendiconti della LXX Riunione Annuale AEI, paper 1-4-01, Rimini, Italy, 1969.

[2] G. Basile and G. Marro, Controlled and Conditioned Invariants in Linear System Theory, Prentice Hall, New Jersey, 1992.

[3] G. Basile, G. Marro and A. Piazzi, "A new solution to the disturbance localization problem with stability and its dual," Proceedings of the '84 International AMSE Conference on Modelling and Simulation, vol. 1.2, pp. 19-27, Athens, 1984.

[4] D. Hrovat, "Optimal active suspension structures for quarter-car vehicle models," Automatica, vol. 26 , no. 5 pp. 845-860, 1990.

[5] G. Marro and Barbagli F. "The algebraic output feedback in the light of dual-lattice structures," in Proc. 6th IEEE Mediterranean Conf. on Contr. and Sys., Italy, June 1998.

[6] A. Piazzi and G. Marro, "The role of invariant zeros in multivariable system stability," Proceedings of the 1991 European Control Conference, Grenoble, 1991.

[7] D. Prattichizzo, P. Mercorelli, A. Bicchi, A. Vicino, "Disturbance decoupling control of vehicles with active suspensions", Proc. 1998 IEEE Int. Conf. on Control Applications, 1998. Trieste, Italy.

[8] H.H. Rosenbrock, State-space and multivariable theory, Wiley, New York, 1970.

[9] J.C. Willems and C. Commault, "Disturbance decoupling by measurement feedback with stability or pole placement," SIAM J. Contr. Optimiz., vol. 19, no. 4, pp. 490-504, 1981.

[10] W.M. Wonham and A.S. Morse, "Decoupling and pole assignment in linear multivariable systems: a geometric approach," SIAM J. Control, vol. 8, no. 1, pp. 1-18, 1970. 